\title{
Épidémiologie du VIH/sida et des autres infections sexuellement transmissibles chez les femmes
}

\author{
Florence LOT ${ }^{1}$
}

\begin{abstract}
Résumé
Les femmes représentent presque la moitié des personnes vivant avec le VIH dans le monde et plus de la moitié en Afrique sub-saharienne, en raison d'une transmission hétérosexuelle qui y est prédominante. La féminisation de l'épidémie se poursuit au niveau mondial et en particulier dans les pays où l'épidémie est plus récente (Asie, Amérique latine, Europe de l'Est). En Europe de l'Ouest et en France, cette féminisation semble se stabiliser et les femmes représentaient respectivement $35 \%$ et $38 \%$ des nouveaux cas d'infection par le VIH en 2005. En France, la moitié de ces femmes sont de nationalité d'un pays d'Afrique subsaharienne.

Si l'intrication entre le VIH/sida et les autres infections sexuellement transmissibles est désormais bien documentée, les données épidémiologiques disponibles sur celles-ci sont plus parcellaires. Chez les femmes, la maladie sexuellement transmissible bactérienne la plus courante est l'infection à Chlamydia trachomatis. En France, sa fréquence semble avoir augmenté depuis la fin des années 1990, ce qui doit inciter à promouvoir son dépistage et à rester vigilant par rapport aux autres maladies sexuellement transmissibles, dont la recrudescence n'a pour l'instant concerné que les hommes.
\end{abstract}

Mots clés : VIH, sida, infections sexuellement transmissibles, épidémiologie, surveillance, femmes.

Les femmes ont progressivement pris une place de plus en plus importante dans l'épidémie de VIH/sida, cette féminisation étant observée dans tous les pays, et de façon plus marquée dans ceux où la transmission hétérosexuelle est prédominante. La place croissante des femmes dans les autres maladies sexuellement transmissibles est sans doute aussi une réalité, mais est plus difficile à objectiver, en raison de données plus récentes et plus parcellaires.

Cet article a pour objet de décrire les évolutions et la situation épidémiologique actuelle des femmes face aux infections transmises lors de rapports sexuels $(\mathrm{VIH} / \mathrm{sida}$ et autres maladies sexuellement transmissibles).

Institut national de veille sanitaire, Saint-Maurice, France.
Après un rapide aperçu des circonstances de vulnérabilité des femmes en matière de transmission sexuelle, une deuxième partie aura trait à l'épidémiologie du $\mathrm{VIH} / \mathrm{sida}$ chez les femmes au plan mondial, avec un éclairage particulier sur l'Europe de l'Ouest, puis sur la situation française. Enfin, un point sera fait sur les données disponibles sur les autres maladies sexuellement transmissibles chez les femmes, selon le même découpage géographique.

\section{Vulnérabilité des femmes face aux infections sexuellement transmissibles}

La vulnérabilité des femmes a surtout été décrite par rapport au risque d'infection par le VIH, mais elle existe pour l'ensemble des maladies sexuellement transmissibles.

Les femmes sont plus vulnérables face au VIH au cours des rapports sexuels que les hommes, en raison de plusieurs facteurs biologiques : les zones de muqueuses susceptibles d'être exposées sont tout d'abord plus étendues chez les femmes ; une plus grande quantité de liquides sexuels est transférée par l'homme et la charge virale dans le liquide séminal est plus importante que dans le liquide vaginal; de plus, des microdéchirures des tissus du vagin (ou du rectum) peuvent être causées par la pénétration sexuelle [1]. Enfin, le fait que les maladies sexuellement transmissibles soient souvent asymptomatiques chez les femmes, alors qu'elles augmentent le risque de transmission du VIH, est un facteur supplémentaire de susceptibilité particulière des femmes vis-à-vis du VIH.

A cette vulnérabilité biologique des femmes se surajoute une vulnérabilité socio-économique, qui se caractérise tout d'abord par des difficultés d'accès à l'information et à la prévention, mais également par des difficultés dans la négociation de la prévention avec les partenaires - voire des rapports sexuels forcés - et, enfin, par un moindre accès aux traitements quand ceux-ci sont disponibles.

L'ignorance concernant la sexualité dans sa globalité ou spécifiquement sur le VIH est très répandue chez les femmes. Dans de nombreux pays, une proportion importante de jeunes femmes 
ne savent pas comment se protéger de l'infection. Dans des enquêtes conduites dans plusieurs pays comme le Cameroun, le Mali, le Sénégal ou le Vietnam, au moins deux tiers des femmes âgées de 15 à 24 ans ne pouvaient pas citer trois méthodes de prévention du VIH [2].

L'équilibre des forces dans de nombreux couples, qui penche du côté de l'homme, fait que les femmes n'ont souvent pas le pouvoir de s'abstenir de rapports sexuels ou de demander l'utilisation du préservatif, même si elles soupçonnent que l'homme a eu d'autres partenaires sexuels et pourrait être infecté par le VIH. Une étude en Zambie a, par exemple, montré que seules $11 \%$ des femmes estimaient être en droit de demander à leur conjoint d'utiliser un préservatif [2]. Et dans bien des pays, les normes de genre permettent aux hommes d'avoir plus de partenaires sexuels que les femmes [1].

La violence faite aux femmes est un fléau mondial. Plusieurs études ont montré une forte corrélation entre les abus sexuels à l'encontre des femmes et leur risque d'être infectées par le VIH. La forme de violence la plus fréquente est celle imputable à un partenaire intime. Entre un tiers et la moitié des femmes interrogées en Namibie, au Pérou et en Thaïlande, par exemple, ont affirmé avoir été agressées physiquement et/ou sexuellement par leurs partenaires. Or, les femmes n'ont souvent aucun recours juridique dans des pays où les lois destinées à prévenir les violences conjugales n'existent pas ou sont mal appliquées [2].

En termes d'accès aux soins, les femmes ont généralement encore un moindre accès à une prise en charge et aux traitements antirétroviraux que les hommes, notamment lorsque ces traitements sont dispensés par le secteur privé ou dans le cadre d'essais cliniques [2].

Les stratégies actuelles de prévention (abstinence, fidélité mutuelle et utilisation du préservatif) ne permettent pas aux femmes de se protéger contre le VIH, d'où l'importance de développer des méthodes de prévention que celles-ci puissent contrôler.

Le préservatif féminin représente une alternative, mais exige toujours une part de négociation et la coopération de l'homme. II est aussi beaucoup plus coûteux que le préservatif masculin. Pas encore commercialisé à grande échelle, il reste ni largement disponible, ni accepté sur le plan social.

Les microbicides viennent s'ajouter et non se substituer aux autres options de prévention. Appliqués dans le vagin, ils ont pour but de réduire le risque de transmission du VIH pendant le rapport sexuel. Mais les résultats récents d'essais d'efficacité à grande échelle, menés en Afrique et en Asie, sont décevants : deux essais utilisant le sulfate de cellulose ont dû être interrompus prématurément début 2007 devant l'observation, dans l'un de ces essais, d'un nombre plus élevé d'infections par le VIH dans le groupe utilisant le microbicide que dans le groupe placebo [3]. Trois autres études sur d'autres produits sont néanmoins toujours en cours dans différents pays d'Afrique, avec des résultats attendus entre fin 2007 et 2009.

Dans ce contexte difficile pour les femmes, l'Onusida a lancé au début de l'année 2004 la " Coalition mondiale sur les femmes et le sida ", un mouvement composé d'associations de la société civile, de réseaux de femmes vivant avec le $\mathrm{VIH}$, de gouvernements et d'organisations des Nations Unies [4]. Cette nouvelle initiative vise à inciter à des actions concrètes pour réagir de manière efficace face aux principaux facteurs de vulnérabilité des femmes.

\section{Épidémiologie du VIH/sida chez les femmes...}

\section{... au plan mondial...}

À l'échelle mondiale, un peu moins de la moitié (48\%) de toutes les personnes vivant avec le VIH sont de sexe féminin (17,7 millions fin 2006 sur un total de 37,2 millions d'adultes) [5]. Ces 17,7 millions de femmes constituent une augmentation de plus d'un million par rapport à 2004 .

C'est dans les régions où les rapports hétérosexuels sont un mode de transmission prédominant du VIH, comme en Afrique subsaharienne et aux Caraïbes, que l'épidémie touche le plus gravement les femmes. Les femmes et les jeunes filles représentent $59 \%$ des adultes vivant avec le VIH en Afrique subsaharienne (figure 1). Dans les Caraïbes, au Moyen-Orient, en Afrique du Nord et en Océanie, près d'un adulte sur deux vivant avec le VIH est une femme.

Les femmes jeunes, entre 15 et 24 ans, sont particulièrement vulnérables, en raison de la fréquence, dans de nombreux pays, de rapports sexuels avec des hommes beaucoup plus âgés, plus susceptibles d'être infectés par le VIH. Le fait qu'il s'agisse souvent des premiers rapports sexuels chez ces femmes augmente encore le risque de transmission du VIH. En Afrique sub-saharienne, les jeunes femmes auraient un risque d'être infectées par le VIH trois fois plus élevé que celui des hommes du même âge. En Afrique du sud, qui reste l'épicentre de l'épidémie mondiale, la prévalence chez les femmes de 15 à 24 ans serait même quatre fois supérieure à celle chez les jeunes hommes $(17 \%$ versus $4,4 \%$ en 2005) [5]. En Afrique de l'Est, où la propagation du VIH a plutôt tendance à décroître, les différences de prévalence entre femmes et hommes sont moins marquées (exemple de l'Ouganda en $2005: 8 \%$ chez les femmes versus $5 \%$ chez les hommes). En Afrique occidentale et centrale, les prévalences chez les femmes enceintes restent moindres, plutôt stables, voire en diminution, ce qui n'exclut pas des épidémies croissantes dans certains pays (au Mali par exemple, la prévalence du VIH est passée de 2,5\% en 2002 à $3,4 \%$ en 2005 chez les femmes de 15 à 19 ans) [5].

Dans de nombreux pays d'Asie, d'Europe de l'Est et d'Amérique latine, la proportion de femmes vivant avec le VIH continue de croître (figure 1), au fur et à mesure que le VIH se propage des 
Figure 1

Pourcentage de femmes adultes (15-49 ans) parmi les personnes vivant avec le VIH dans le monde et dans les régions

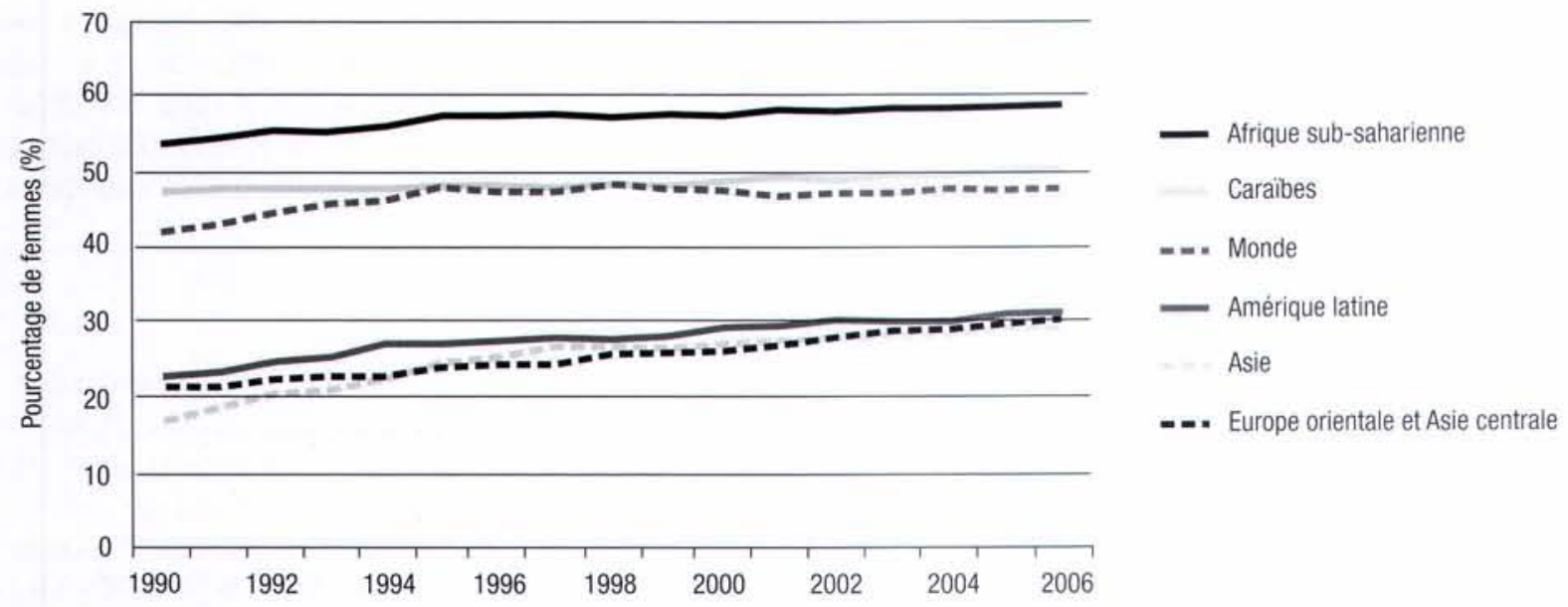

Source : Onusida/OMS, 2006.

Figure 2

Proportion de femmes parmi les diagnostics d'infection par le VIH, en 2005, en Europe de l'Ouest

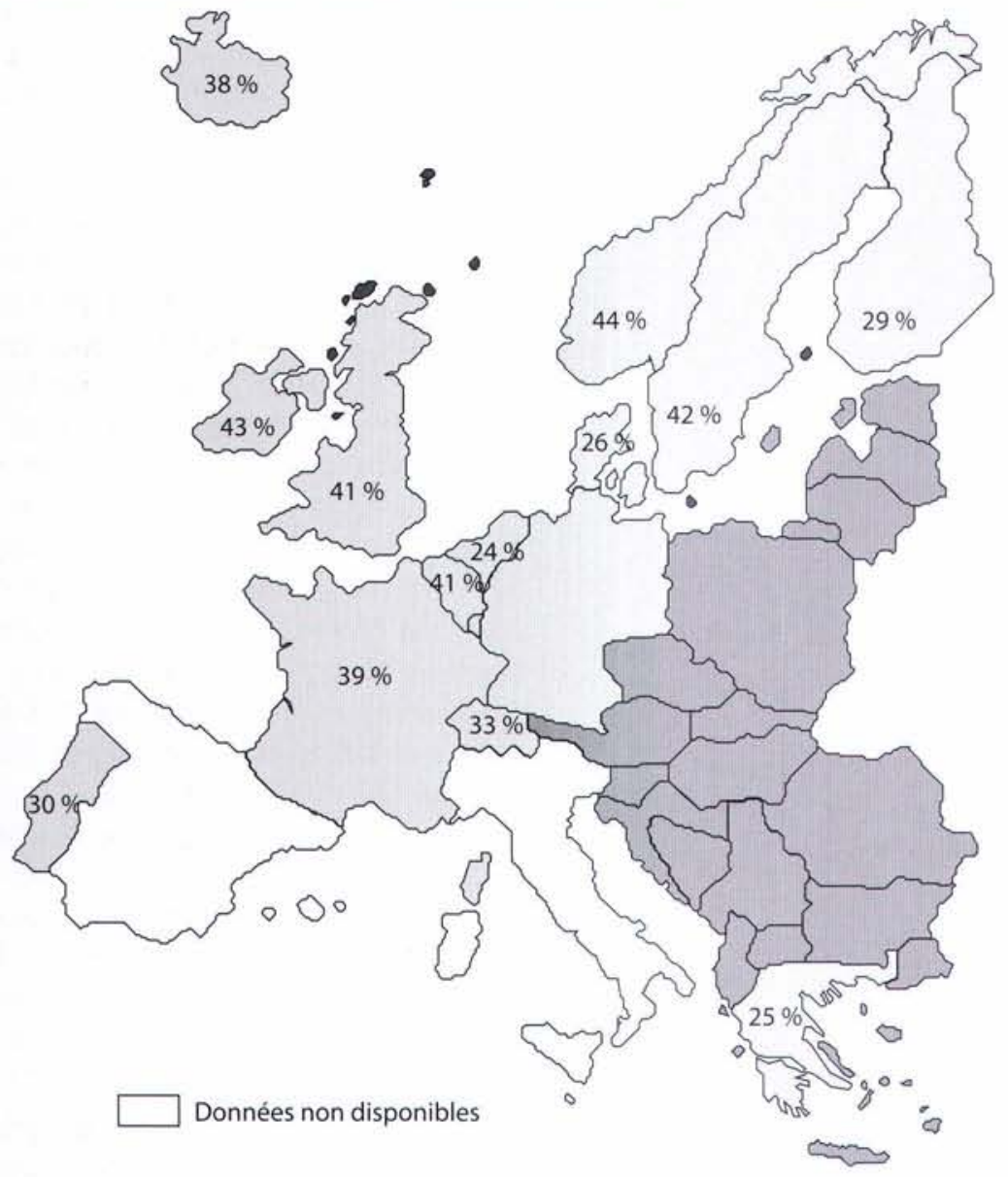

Source : EuroHIV, données au 31/12/2005. 
populations particulièrement exposées à d'autres populations moins exposées.

En Asie, il semble qu'une forte proportion de femmes séropositives aient été contaminées par leur partenaire régulier, lui-même contaminé lors de rapports sexuels rémunérés (la prévalence du $\mathrm{VIH}$ chez les professionnelles du sexe pourrait atteindre un niveau extrêmement élevé, de l'ordre de $50 \%$ dans certains états de I'Inde, par exemple). En Europe de l'Est, une grande proportion de femmes a été contaminée lors de rapports sexuels avec un usager de drogues. En Amérique latine, un nombre croissant de femmes contractent le VIH auprès de leurs partenaires masculins, eux-mêmes contaminés lors de rapports sexuels avec des professionnelles du sexe et de rapports sexuels non protégés entre hommes.

\section{... en Europe de l'Ouest...}

Même si la situation des femmes vis-à-vis du VIH est beaucoup moins problématique en Europe de l'Ouest, grâce notamment à un accès plus large à l'information, à la prévention et aux traitements antirétroviraux, la transmission du VIH se poursuit dans l'ensemble des pays de cette zone géographique.

En Europe de l'Ouest, 25928 nouveaux diagnostics d'infection par le VIH ont été rapportés en 2005, soit un taux de 85 par million d'habitants [6]. Le nombre de diagnostics notifiés chaque année a augmenté dans les deux sexes entre 1997 et 2004, mais de façon plus marquée chez les femmes ${ }^{2}$. La proportion de femmes est donc passée de $26 \%$ en 1997 à $36 \%$ en 2004 (puis $35 \%$ en 2005). Cette augmentation a particulièrement concerné les femmes âgées de 40 à 49 ans, ainsi que les femmes contaminées par rapports hétérosexuels et celles originaires d'Afrique subsaharienne (communication personnelle, EuroHIV).

En 2005, la proportion de femmes varie d'un pays à l'autre, de $20 \%$ en Allemagne à $44 \%$ en Norvège (figure 2), ces différences étant essentiellement expliquées par une distribution globale différente des modes de transmission. En Allemagne, le nombre de diagnostics de VIH chez les homosexuels est presque le double de celui chez les hétérosexuels. En Norvège et dans les autres pays où le pourcentage de femmes parmi les diagnostics de VIH est proche de $40 \%$, on compte au contraire un nombre de personnes contaminées par rapports hétérosexuels bien supérieur à celui des personnes contaminées par rapports homosexuels.

Dans la majorité des pays d'Europe de l'Ouest, la proportion de femmes d'Afrique sub-saharienne est importante. Elle varie en 2005 de $17 \%$ au Portugal à $65 \%$ en Suède (figure 3).

Comme témoins de la prévalence du VIH dans la population hétérosexuelle active, les femmes enceintes constituent un groupe intéressant pour recueillir des données sur la proportion de personnes infectées par le VIH. Plusieurs pays d'Europe de l'Ouest

\footnotetext{
2 Évolutions analysées pour les pays disposant de données sur l'ensemble de la période.
}

disposent de telles données et les taux de prévalence varient de $0,3 \%$ en Irlande en 2002-2003 à moins de $0,03 \%$ en Finlande en 2004 [7]. Des taux intermédiaires sont retrouvés en Allemagne, Italie, Espagne, Royaume-Uni, Pays-Bas et Norvège. Au Royaume-Uni, les plus fortes prévalences sont retrouvées chez les femmes nées en Afrique sub-saharienne (2,2\% en 2004), comparativement à une prévalence de $0,07 \%$ chez celles nées dans le pays.

\section{... en France}

En France, les dernières données de prévalence disponibles chez les femmes enceintes datent de 1997 (enquête Prevagest) [8]. Le taux de prévalence du VIH chez les femmes enceintes de la région parisienne était environ huit fois plus élevé chez les femmes nées en Afrique sub-saharienne par rapport à celles nées en France métropolitaine (2,2\% versus $0,28 \%)$. Chez les femmes nées dans les Caraïbes, y compris dans les départements français d'Amérique, le taux de prévalence était de $0,83 \%$ (non significativement différent de celui observé chez les femmes nées en métropole).

\section{Surveillance du sida}

Pour analyser la situation actuelle des femmes en France visà-vis du VIH/sida, plusieurs types de données existent. La surveillance du sida montre que les femmes ont été moins touchées que les hommes depuis le début de l'épidémie, puisqu'elles concentrent un total d'environ 12300 cas de sida cumulés pour plus de 49000 chez les hommes. En revanche, l'observation des tendances au cours des 20 dernières années met nettement en évidence une augmentation de la proportion de femmes diagnostiquées chaque année, qui atteint $33 \%$ en 2005 (figure 4). On peut néanmoins noter que le nombre de cas de sida est relativement stable chez les femmes depuis 1997, autour de 400 à 500 cas annuels. Cette apparente stabilité chez les femmes est en fait la résultante de deux tendances inverses : une diminution du nombre de cas de sida chez les femmes françaises et une augmentation chez les femmes étrangères entre 1998 et 2002 (figure 5). Depuis 2001, plus d'un cas sur deux est diagnostiqué chez une femme de nationalité étrangère. L'augmentation du nombre de cas de sida la plus importante a concerné les femmes d'Afrique sub-saharienne (de 89 cas en 1998 à 198 cas en 2002), en particulier du Cameroun, de Côte d'Ivoire et du CongoBrazzaville, et dans une moindre mesure les femmes haïtiennes (de 14 à 24 cas) (figure 6). Ces augmentations sont concordantes avec un accroissement des flux migratoires en provenance de ces pays et les niveaux de prévalence du VIH élevés qui y sont retrouvés ( $11 \%$ en Côte d'Ivoire, $8 \%$ au Cameroun et $6 \%$ au Congo Brazzaville, en 1999) [9].

Depuis 2002, le nombre de cas de sida a diminué chez les femmes d'Afrique sub-saharienne, quelle que soit leur nationalité, à l'exception des femmes camerounaises. Sur la même 
Figure 3

Proportion de femmes originaires d'Afrique sub-saharienne parmi les diagnostics d'infection par le VIH chez les femmes en 2005 en Europe de l'Ouest

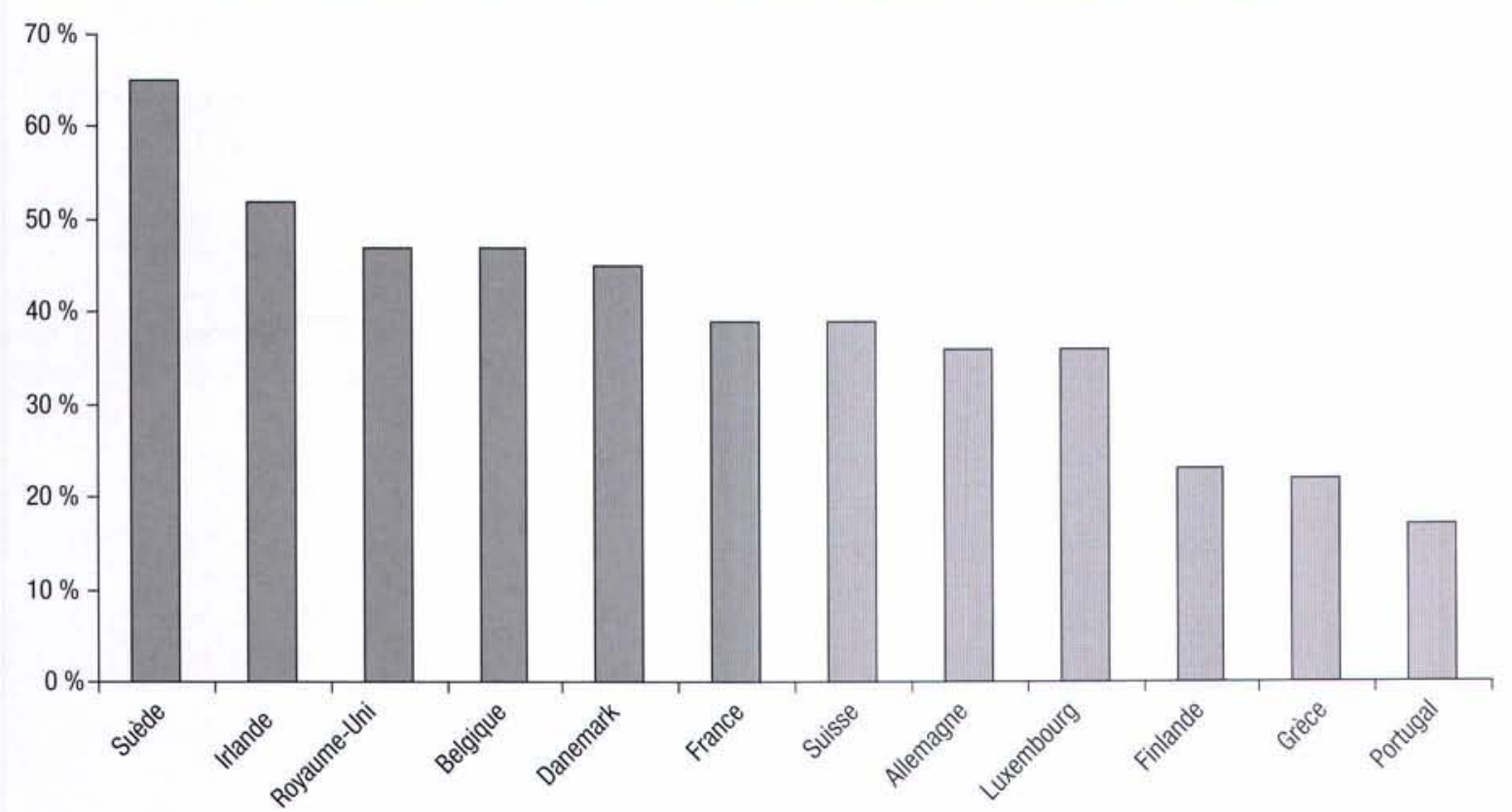

Source : EuroHIV, données au 31/12/2005.

Figure 4

Nombre de cas de sida selon le sexe et proportion de cas féminins

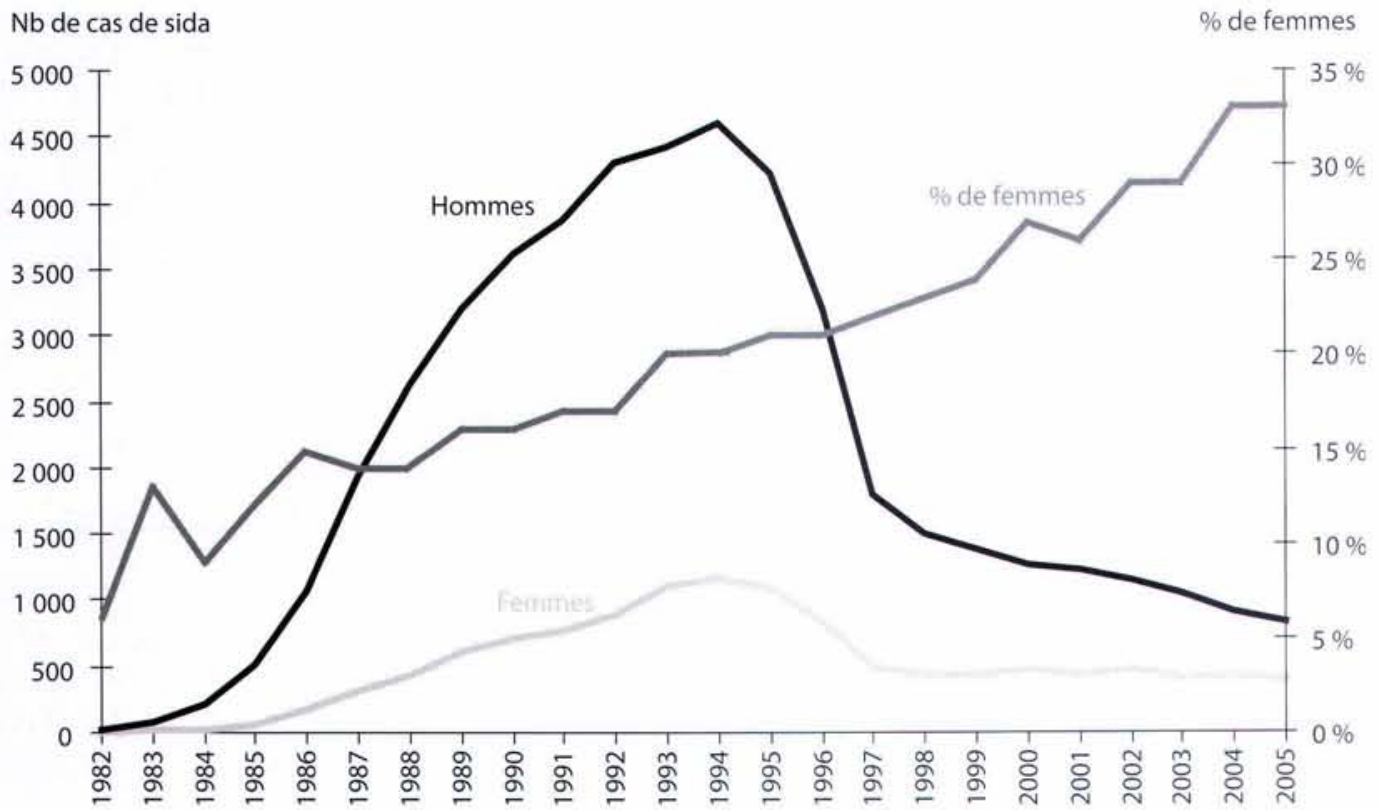

Année de diagnostic

Source : InVS, données au 30/06/2006, redressées pour les délais de déclaration. 
Figure 5

Nombre de cas de sida chez les femmes de nationalité française et étrangère

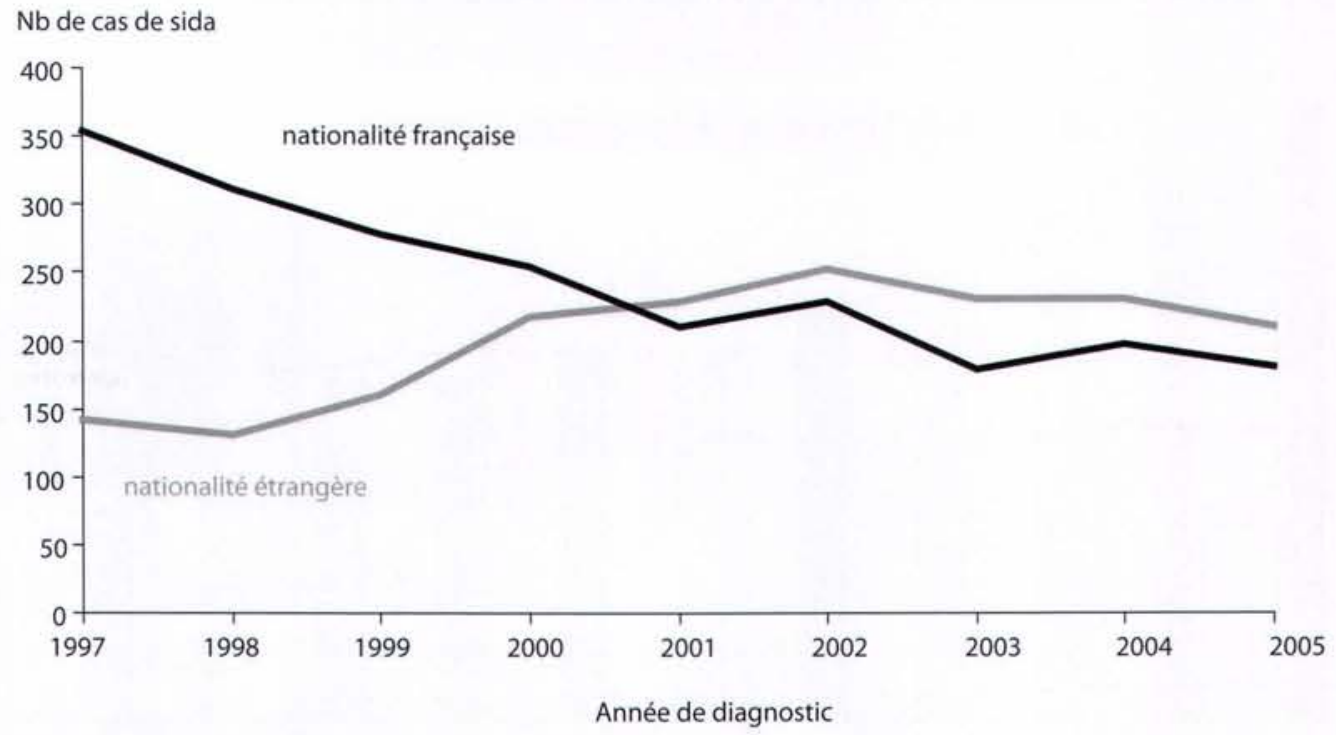

Source : InVS, données au 30/06/2006, redressées pour les délais de déclaration.

Figure 6

Nombre de cas de sida chez les femmes de nationalité étrangère

$\mathrm{Nb}$ de cas de sida

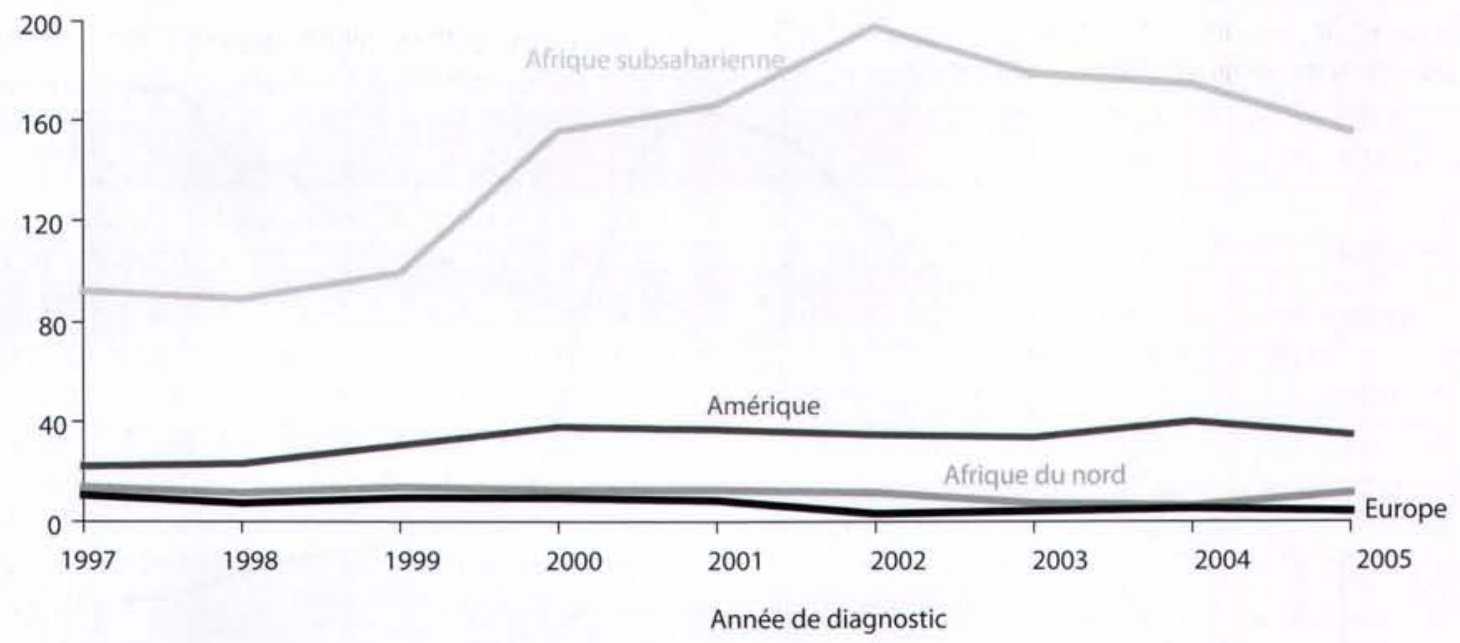

Source : InVS, données au 30/06/2006, redressées pour les délais de déclaration. 
Figure 7

Nombre de découvertes de séropositivité au VIH selon le sexe, le mode de contamination et la nationalité

$\mathrm{Nb}$ de diagnostics $\mathrm{VIH}$

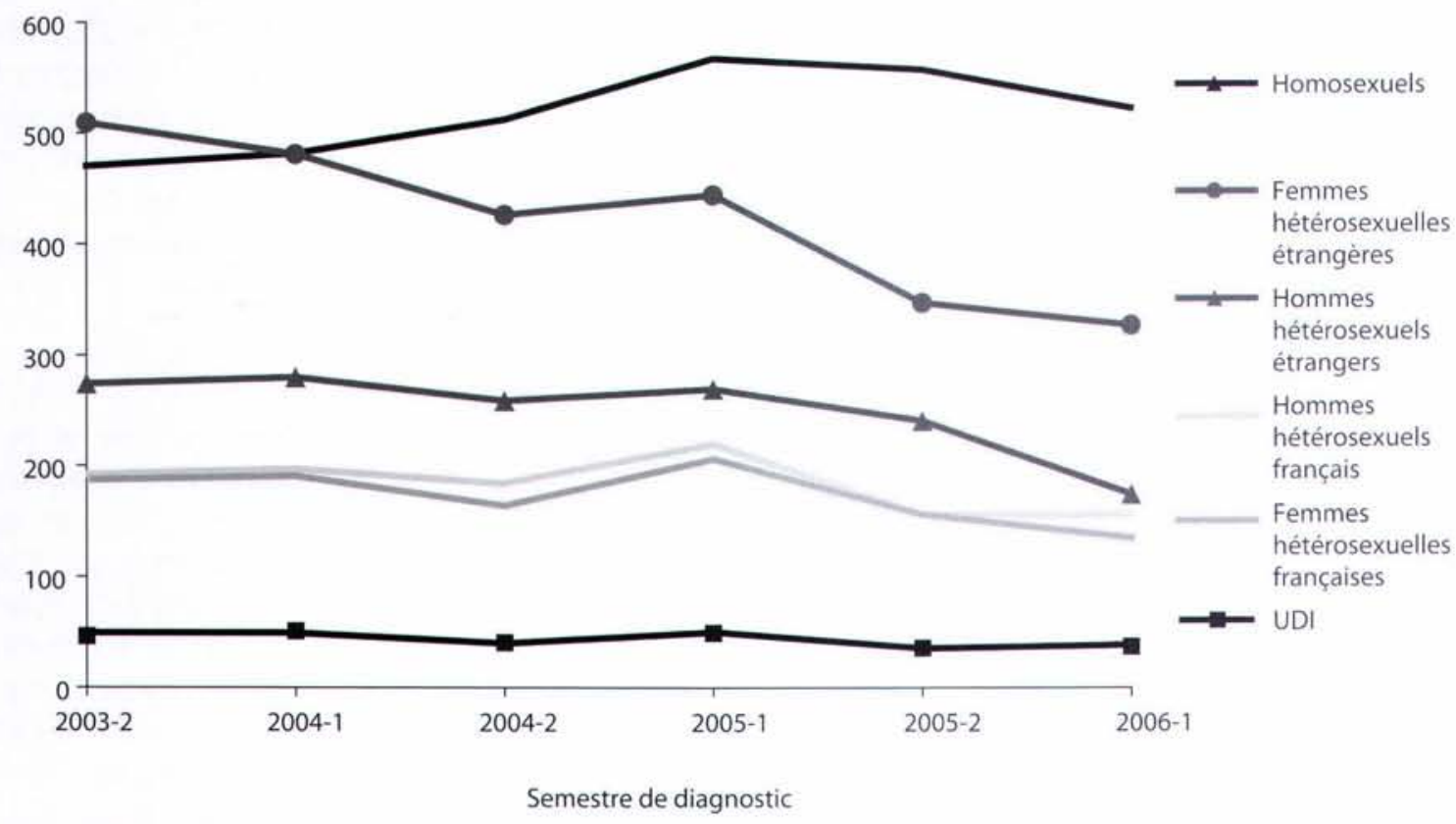

Source : InVS, données au 30/06/2006, redressées pour les délais de déclaration.

période, les flux migratoires en provenance des pays d'Afrique sub-saharienne ont pourtant globalement continué à augmenter, notamment en provenance du Cameroun, de la Côte d'lvoire et du Mali. En revanche, ils sont stables pour le Congo-Brazzaville (source Ined, données jusqu'en 2004). Les évolutions entre le nombre de cas de sida et les flux migratoires sont donc moins parallèles sur cette période récente.

\section{Déclaration obligatoire du VIH}

Les données de la déclaration obligatoire du VIH confortent celles de la surveillance du sida, tout en témoignant d'une proportion encore plus importante de femmes. En 2005, elles représentent $38 \%$ des découvertes de séropositivité. En termes de tendances, le nombre de femmes ayant découvert leur séropositivité diminue depuis 2003, de façon comparable chez les femmes de nationalité étrangère et celles de nationalité française (figure 7). La diminution observée chez les femmes étrangères est surtout liée à une diminution chez les africaines, mais s'observe aussi parmi les autres nationalités.

En 2005, les femmes d'Afrique sub-saharienne représentaient environ la moitié des découvertes de séropositivité chez les femmes (figure 8), soit un nombre proche de 750 . Par ordre décroissant, il s'agit essentiellement de femmes camerounaises $(n=239)$, de Côte d'Ivoire $(n=160)$, du Congo-Brazzaville
( $n=88)$, du Mali $(n=51)$ et du Togo $(n=28)$. Les autres pays d'Afrique comptabilisent chacun moins de 20 diagnostics d'infection par le VIH chez des femmes.

Un quart des femmes ayant découvert leur séropositivité en 2005 est de nationalité française, ce qui représente environ 400 femmes.

Les femmes d'une autre nationalité se répartissent ainsi : $86 \mathrm{du}$ continent américain (dont 55 d'Haïti), 22 d'Afrique du Nord, 15 d'Asie (dont huit de Thaillande), 11 d'Europe de l'Ouest (dont huit du Portugal) et dix d'Europe de l'Est ou centrale.

Les principales caractéristiques des femmes ayant découvert leur séropositivité au VIH en 2005 sont précisées dans le tableau I. En raison d'un effectif limité, les femmes de nationalité étrangère autre que celles d'un pays d'Afrique sub-saharienne ont été regroupées. Quelle que soit leur nationalité, elles ont été contaminées de manière quasi exclusive lors de rapports hétérosexuels, l'usage de drogues par voie intraveineuse concernant moins de $1 \%$ d'entre elles.

Les femmes d'Afrique sub-saharienne ayant découvert leur séropositivité VIH en 2005 sont en moyenne plus jeunes ( 32 ans) que les femmes françaises (38 ans).

Elles semblent plus fréquemment dans une situation de précarité, puisque seules $16 \%$ d'entre elles ont une activité professionnelle (versus $46 \%$ chez les femmes françaises). 
Figure 8

Répartition des 1530 femmes ayant découvert leur séropositivité VIH en 2005, en fonction de leur nationalité (par groupe)

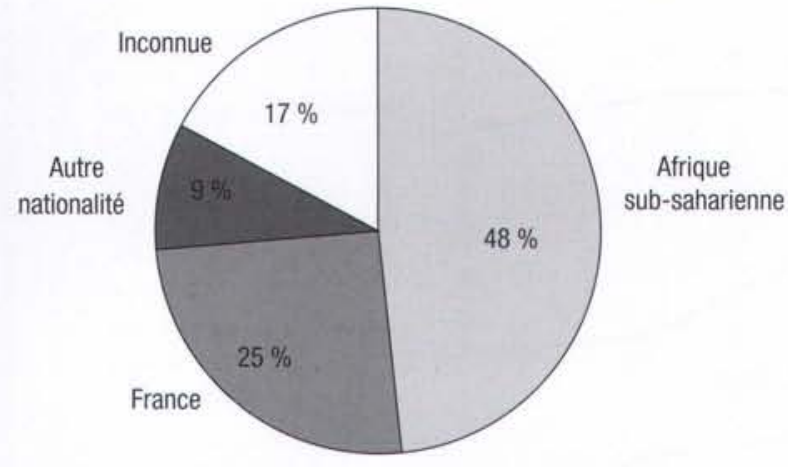

Source : InVS, données au 30/06/2006.

Elles vivent en majorité en île-de-France ( $59 \%)$, tandis que $31 \%$ des femmes françaises y sont domiciliées. Les départements français d'Amérique concentrent aussi $11 \%$ des femmes françaises.

La grossesse représente une opportunité de diagnostic de l'infection plus fréquente pour les femmes africaines que pour les femmes françaises ( $20 \%$ versus $13 \%$ ).

Au moment de la découverte de leur séropositivité, elles sont plus souvent que les femmes françaises à un stade asymptomatique (68\% versus $59 \%$ ) et moins souvent à un stade de sida ( $11 \%$ versus $17 \%$ ). La proportion de femmes africaines à un stade asymptomatique a tendance à augmenter depuis 2003 (de $61 \%$ à $68 \%, p=0,004$ ) et inversement celles au stade sida diminuent (de $16 \%$ à $11 \%, p=0,009$ ). En revanche, aucune évolution significative en termes de stade clinique n'est retrouvée chez les femmes françaises.

Des précisions sur le partenaire à l'origine probable de la contamination n'ont pu être recueillies que chez un quart des femmes d'Afrique sub-saharienne, mais chez plus de la moitié des femmes françaises. Trois quarts des femmes françaises ont été contaminées par un partenaire stable.

Seules $14 \%$ de femmes d'Afrique sub-saharienne avaient un antécédent de sérologie VIH négative (versus $28 \%$ chez les femmes françaises), mais cette proportion a tendance à augmenter depuis 2003 (de $8 \%$ à $14 \%, p=0,005$ ).

Le délai moyen entre contamination et diagnostic est connu chez une très faible proportion de femmes africaines $(7 \%)$ et, lorsqu'il est connu, il est beaucoup plus long que chez les femmes françaises (37,2 mois versus 13,4 mois). Les résultats du test d'infection récente sont aussi en faveur d'une contamination des femmes africaines plus ancienne, puisque seules $8,5 \%$ ont été infectées dans les six mois précédant le diagnostic du VIH (contre $29 \%$ des femmes françaises).
Un quart des femmes d'Afrique sub-saharienne ont été contaminées par un virus $\mathrm{VIH}-1$ de sous-type $B$, sous-type très peu présent en Afrique et au contraire majoritaire en France et en Europe. Le fait qu'une proportion croissante de femmes ait été contaminée par ce sous-type entre 2003 et 2005 (de $17 \%$ à $25 \%, p=0,0003$ ) est plutôt en faveur d'une augmentation de la proportion de contaminations sur le territoire français. À l'inverse, un tiers des femmes françaises a été infecté par un virus de sous-type non B, prédominant en Afrique. Dans la moitié des cas, ces femmes sont nées en Afrique sub-saharienne et/ou ont été contaminées par un partenaire africain.

\section{Données de dépistage}

Les données de dépistage, au travers du suivi de l'activité de dépistage ou des enquêtes en population générale, complètent la description de la situation des femmes vis-à-vis du VIH. En France, I'offre de dépistage du VIH est très large, en raison des nombreuses structures qui existent pour se faire dépister. Parmi les 3,5 millions de tests réalisés en 1997, $59 \%$ concernaient des femmes. Depuis cette date, l'activité de dépistage a progressivement augmenté et en 2005, plus de 5 millions de tests ont été réalisés (mais la part des femmes est inconnue) [10].

Les femmes sont néanmoins toujours plus testées que les hommes, comme le montrent les données de l'enquête KABP 2004 [11]. Les femmes déclarent presque deux fois plus souvent que les hommes avoir été dépistées au cours de la dernière année ( $14,0 \%$ versus $7,2 \%$ ), cette différence s'expliquant notamment par la proposition de dépistage faite lors des consultations prénatales et lors des grossesses. Plus les femmes sont jeunes, plus la probabilité qu'elles aient fait un test dans les douze derniers mois est forte. II en est de même si elles habitent en zone urbaine, si elles sont multipartenaires ou si elles ont utilisé un préservatif dans les 12 derniers mois.

A cette offre de dépistage très large, à laquelle les femmes répondent le plus souvent en se rendant dans un laboratoire de ville avec une prescription médicale (pour environ deux tiers des femmes testées), s'ajoute la possibilité de se faire dépister dans un centre de dépistage anonyme et gratuit. Néanmoins, en 2004 , seules $5,1 \%$ des femmes avaient effectué leur dernier test de dépistage dans une telle structure (et 6,3\% des hommes) [11]. Parmi les consultants de ces centres, la proportion de femmes a fortement augmenté entre 1988 et 1996 (de $36 \%$ à $46 \%$ ) mais est restée ensuite constante jusqu'en 2005, alors que le nombre de sérologies réalisées continue d'augmenter [12]. La proportion de tests positifs chez les femmes a toujours été inférieure à celle des hommes, mais la différence s'est considérablement réduite au cours des années. En 2005, cette proportion est de 3 pour mille chez les femmes testées en centre de dépistage anonyme et gratuit, contre 4,5 pour mille chez les hommes. 
Tableau 1

Description des femmes âgées de 15 ans et plus ayant découvert leur séropositivité au VIH en 2005 en fonction de leur groupe de nationalité ${ }^{1}$

\begin{tabular}{|c|c|c|c|}
\hline & Française & $\begin{array}{l}\text { D'un pays d'Afrique } \\
\text { sub-saharienne }\end{array}$ & Autre nationalité \\
\hline & $\mathrm{n}=397$ & $n=759$ & $n=144$ \\
\hline \multicolumn{4}{|l|}{ Âge (années) } \\
\hline Moyen & 38,4 & 32,3 & 36,5 \\
\hline Médian [p. 25-p75] & 37 [28-49] & $30[27-36]$ & $34[29-43]$ \\
\hline En activité professionnelle (\%) & 46,4 & 16,1 & 26.4 \\
\hline \multicolumn{4}{|l|}{ Région de domicile (\%) } \\
\hline Île-de-France & 30,5 & 59,2 & 35,4 \\
\hline PACA & 8,1 & 2,9 & 2,8 \\
\hline Rhône-Alpes & 8,3 & 5,8 & 2,8 \\
\hline Départements français d'Amérique & 11,3 & 0,3 & 34,7 \\
\hline Autre/inconnu & 41,8 & 31,9 & 24,3 \\
\hline \multicolumn{4}{|l|}{ Motif du dépistage (\%) } \\
\hline Signes cliniques ou biologiques & 31.7 & 24,4 & 36,1 \\
\hline Grossesse & 13,4 & 19,7 & 19,4 \\
\hline Exposition à risque & 21.9 & 15,8 & 16,7 \\
\hline Bilan & 14,9 & 14,0 & 13,2 \\
\hline Autre/inconnu & 18,1 & 26,1 & 14,6 \\
\hline \multicolumn{4}{|l|}{ Stade clinique de l'infection (\%) } \\
\hline Primo-infection & 8,6 & 1,4 & 2,8 \\
\hline Asymptomatique & 59.4 & 68,0 & 52,1 \\
\hline Symptomatique non sida & 10,3 & 12,9 & 15,3 \\
\hline Sida & 16,9 & 11,1 & 22,2 \\
\hline Inconnu & 4,8 & 6,6 & 7,6 \\
\hline \multicolumn{4}{|l|}{ Mode de contamination probable (\%) } \\
\hline Hétérosexuel & 86,7 & 83,8 & 83,3 \\
\hline Usage de drogues en intraveineux & 0,8 & 0,4 & 0,7 \\
\hline Transfusion & 0,2 & - & - \\
\hline Inconnu & 12,3 & 15,8 & 16,0 \\
\hline Précisions disponibles sur le partenaire sexuel (\%) & 57 & 26 & 43 \\
\hline \multicolumn{4}{|l|}{ Partenaire sexuel ${ }^{2}$} \\
\hline Stable & 75,9 & 62,2 & 82,4 \\
\hline Occasionnel & 20,0 & 19,5 & 15,7 \\
\hline Anonyme & 1,0 & 7,3 & 1,9 \\
\hline Info non disponible & 3,1 & 11,0 & - \\
\hline Antécédent d'une sérologie négative (\%) & 27,5 & 13,7 & 13,9 \\
\hline Estimation possible d'une date de contamination (\%) & 22,7 & 7,4 & 7,6 \\
\hline Délai moyen entre dates de contamination et de diagnostic ${ }^{3}$ (mois) & 13,4 & 37,2 & 23,8 \\
\hline Infection $\leq 6$ mois $(\%)$ & 29,0 & 8,5 & 10,9 \\
\hline \multicolumn{4}{|l|}{ Type de virus (\%) } \\
\hline $\mathrm{VIH}-1$ & 95,5 & 93,4 & 97,9 \\
\hline $\mathrm{VIH}-2$ & 0,8 & 2,4 & 0,7 \\
\hline $\mathrm{VIH}-1+2$ & 1.7 & 1,8 & 0,7 \\
\hline Inconnu & 2,0 & 2,4 & 0,7 \\
\hline \multicolumn{4}{|l|}{ Sérotypage (\%) } \\
\hline B & 66,7 & 25,4 & 74,0 \\
\hline Non B & 33,3 & 74,6 & 26,0 \\
\hline
\end{tabular}

${ }^{1}$ Sont exclues les 260 femmes de nationalité inconnue.

2 Parmi les femmes pour lesquelles on dispose de précisions sur le partenaire sexuel.

3 Parmi les femmes pour lesquelles une date de contamination a pu être estimée

Source InVS, données au 30/06/2006. 


\section{Épidémiologie des autres infections sexuellement transmissibles chez les femmes...}

La présence d'une infection sexuellement transmissible non traitée, par l'inflammation ou les ulcérations qu'elle provoque, augmente le risque de transmission du VIH lors d'un rapport sexuel non protégé. II semble que ce soit plus particulièrement vrai des infections ulcératives, qui multiplieraient le risque de transmission du VIH par un facteur dix. À l'inverse, la prévention et le traitement de ces maladies réduisent le risque de transmission du $\mathrm{VIH}$ par voie sexuelle, en particulier dans les populations ayant un grand nombre de partenaires sexuels, comme les professionnels du sexe et leurs clients [13].

Les maladies sexuellement transmissibles ont aussi des répercussions importantes en termes de morbidité et de mortalité, allant de lésions douloureuses invalidantes pour beaucoup d'entre elles à des atteintes neurologiques dans le cas d'une syphilis non traitée.

Chez les femmes, les maladies sexuellement transmissibles, et notamment les infections à Chlamydia non traitées, peuvent être responsables de stérilité ou de grossesse extra-utérine. Elles entraînent aussi des issues défavorables de la grossesse (fausse couche, accouchement prématuré, décès périnatal, infections chez le nouveau-né). C'est le cas notamment des femmes souffrant d'une gonococcie, d'une chlamydiose non traitée, d'herpès génital ou d'une syphilis précoce. Jusqu'à $35 \%$ des grossesses chez les femmes souffrant d'une gonococcie non traitée se terminent par une fausse couche ou un accouchement pré-terme et jusqu'à $10 \%$ par un décès périnatal. En l'absence de prophylaxie, $30 \%$ à $50 \%$ des nourrissons nés de mères atteintes d'une gonococcie non traitée et jusqu'à $30 \%$ de ceux nés de mères atteintes d'une chlamydiose non traitée contractent une ophtalmie du nouveau-né qui peut conduire à la cécité. À l'échelle mondiale, 1000 à 4000 nouveau-nés perdent la vue chaque année à cause de cette affection. En l'absence de traitement, $25 \%$ des grossesses chez les femmes souffrant d'une syphilis précoce se soldent par le décès de la femme et $1 \%$ par le décès du nouveau-né [13].

Au vu de ces chiffres, les maladies sexuellement transmissibles constituent un problème de santé publique majeur dans le monde. Les stratégies de luttes à la fois préventive et curative doivent donc se poursuivre, afin de réduire les répercussions dramatiques que ces infections entraînent chez les femmes et les nouveau-nés.

\section{... au plan mondial...}

Dans la plupart des pays, les systèmes de surveillance des autres maladies sexuellement transmissibles sont moins bien développés et les données épidémiologiques moins bien connues que celles sur le $\mathrm{VIH} / \mathrm{sida}$. Une des raisons est que les maladies sexuellement transmissibles regroupent des pathologies très hétérogènes en termes d'agents infectieux impliqués, puisque plus de 30 agents pathogènes bactériens, viraux et parasitaires peuvent être sexuellement transmissibles. De cette diversité résulte aussi une hétérogénéité des populations touchées, notamment selon le sexe. Une autre difficulté pour le recueil de données réside dans le fait que les infections sexuellement transmissibles sont souvent asymptomatiques ou font l'objet de symptômes bénins et passagers.

L'OMS estime néanmoins à plus de 340 millions le nombre annuel de nouveaux cas curables (syphilis, gonococcies, infections à Chlamydia trachomatis et trichomonases) dans le monde chez les hommes et femmes âgés de 15 à 49 ans [13]. La plus grande part de ces maladies sexuellement transmissibles est diagnostiquée dans la région de l'Asie du Sud et du Sud-Est, suivie de l'Afrique sub-saharienne, puis de l'Amérique latine et des Caraïbes. En Afrique, la prévalence de la syphilis chez les femmes enceintes serait comprise entre $4 \%$ et $15 \%$.

Des millions de cas d'infections sexuellement transmissibles virales se déclarent aussi tous les ans, les principaux virus étant (en dehors du VIH) les herpes virus humains, les papillomavirus humains et le virus de l'hépatite $B$.

L'infection due au virus de l'herpes simplex type 2 (HSV-2) est la première cause d'ulcérations génitales dans les pays en développement. Partout dans le monde, la séropositivité HSV-2 est plus fréquente chez les femmes que chez les hommes et augmente avec l'âge. En Afrique sub-saharienne, 30 à $80 \%$ des femmes (et 10 à $50 \%$ des hommes) seraient porteurs de ce virus. L'herpes virus joue un rôle important dans la transmission du VIH. Une étude réalisée en Tanzanie a montré que $22 \%$ des infections à VIH chez les femmes étaient attribuables à une infection à HSV-2 (infection pré-existante ou séroconversion à HSV-2) [14]. Une autre étude a montré que le traitement suppressif du HSV-2 réduisait l'excrétion génitale du VIH chez la femme [15].

Le papillomavirus humain cause chaque année environ 500000 cancers du col de l'utérus, recensés pour la plupart dans des pays à faibles ressources, dont la moitié seraient mortels. C'est le cancer le plus fréquent chez la femme, après le cancer du sein.

Le virus de I'hépatite $B$, qui se transmet entre autre par voie sexuelle, cause chaque année 350 millions de cas d'hépatite chronique (hommes et femmes) et au moins un million de décès par cirrhose ou cancer primitif du foie.

\section{... en Europe...}

Au niveau du continent européen, une grande hétérogénéité est retrouvée en ce qui concerne le diagnostic des maladies sexuellement transmissibles et leur traitement, mais aussi dans les systèmes de surveillance, ce qui complique la comparaison des données disponibles. Néanmoins, en dehors de l'Europe de l'Est 
où la dynamique de ces infections (et notamment de la syphilis) est explosive depuis l'effondrement du bloc soviétique, la situation est beaucoup moins alarmante qu'au niveau mondial.

Même si, dans plusieurs états de l'Union européenne, le nombre d'infections à gonocoques a considérablement augmenté au cours des dix dernières années et des épidémies de syphilis rapportées récemment, les femmes sont peu concernées par la recrudescence de ces maladies sexuellement transmissibles, la population la plus touchée étant celle des homosexuels masculins [16].

Les femmes sont surtout concernées par les infections à Chlamydia trachomatis, dont la prévalence est variable mais non négligeable. Elle est maximale chez les femmes jeunes, âgées de 18 à 24 ans : de l'ordre de 2 à $4 \%$ dans les enquêtes nationales européennes en population générale [17]. En termes de tendances, après une forte augmentation des cas détectés, liée à la diffusion de tests de dépistage dans les années 1980, une diminution de l'incidence s'est ensuite amorcée. Cette baisse a cessé au milieu des années 1990 et une recrudescence a été signalée à partir de 2000 dans plusieurs pays d'Europe de l'Ouest, notamment au Royaume-Uni $[17,18]$. Les augmentations les plus importantes ont été observées chez les femmes de 16 à 19 ans. Elles reflètent probablement une aggravation des comportements sexuels à risque, mais peuvent également s'expliquer par un meilleur dépistage grâce à une sensibilisation plus grande du public et des professionnels de santé vis-à-vis des chlamydioses génitales [19].

On note aussi une tendance à l'augmentation d'autres maladies sexuellement transmissibles chez les femmes, comme les gonococcies ou la syphilis, toujours en Angleterre et au Pays de Galles, mais de façon beaucoup moins marquée et à un niveau bien moindre que chez les hommes [18,19]. II est néanmoins très difficile de faire la part d'un dépistage devenu beaucoup plus systématique chez les femmes, alors qu'il est plus ancien et organisé chez les hommes. Quelques cas de syphilis congénitale sont encore néanmoins diagnostiqués chaque année dans la plupart des pays d'Europe de l'Ouest.

En ce qui concerne les infections herpétiques, les niveaux de prévalence varient d'un pays à l'autre. La prévalence des infections à HSV-2 serait plus élevée dans le nord de l'Europe (de 15 à $30 \%$ dans les pays nordiques) qu'en Europe de l'Ouest et du Sud (autour de $5 \%$ au Royaume-Uni) [18].

\section{... En France}

En France, comme dans d'autres pays européens, les infections sexuellement transmissibles ont beaucoup fait parler d'elles ces dernières années, avec la résurgence de pathologies jusqu'alors peu fréquentes. Mais cette résurgence a essentiellement concerné les populations homosexuelles, les femmes étant très peu représentées parmi les cas d'infections telles que la syphilis ou la lymphogranulomatose vénérienne.
Pour ce qui concerne la syphilis, le nombre de cas de syphilis infectieuse déclarés entre 2000 et 2005 à travers un système de surveillance sentinelle a concerné une proportion très faible de femmes (5\%), dont l'âge médian était de 31,5 ans. Les femmes sont diagnostiquées plus tardivement que les hommes, puisque $63 \%$ d'entre elles sont à un stade de syphilis latente précoce, signifiant une infection initiale dans les douze derniers mois (contre $32 \%$ chez les hommes). Le diagnostic chez les hommes se fait plus fréquemment aux stades précédents : syphilis primaire (chancre) ou secondaire (éruption). Le caractère sans doute non représentatif du réseau de surveillance ne permet pas de connaître au mieux la diffusion de la syphilis chez les femmes. Dans des pays voisins, les femmes représentent un pourcentage plus important parmi l'ensemble des cas qu'en France ( $12 \%$ au Royaume-Uni en 2004 et $8 \%$ aux Pays-Bas en 2003) [20].

Le dépistage de la syphilis chez les femmes françaises se fait néanmoins de manière très large, dans le cadre des bilans obligatoires réalisés au cours du premier trimestre de la grossesse.

Grâce au réseau Rénago instauré en 1986 et reposant sur des laboratoires de microbiologie volontaires, les tendances et les principales caractéristiques des infections à gonocoques sont disponibles depuis de nombreuses années. Après une baisse continue entre 1986 et 1995 du nombre de gonocoques isolés par an et par laboratoire, celui-ci augmente globalement depuis 1996 chez les deux sexes, mais de façon plus marquée chez les femmes que chez les hommes (+ $160 \%$ versus $+124 \%$ ) [20]. Chez les femmes, cette augmentation est nette entre 1996 et 1998 et à nouveau entre 2002 et 2005. Elle affecte l'ensemble des régions, mais elle est plus importante en Île-de-France. Le nombre de gonocoques isolés reste néanmoins cinq fois moins important chez les femmes que chez les hommes (en 20042005, 0,25 gonocoques/an/laboratoire chez les femmes versus 2,25 chez les hommes). Environ les deux tiers des femmes ayant développé une infection à gonocoques ont entre 16 et 30 ans.

L'infection à Chlamydia trachomatis est l'infection sexuellement transmissible bactérienne la plus fréquente de la femme. La proportion d'identifications positives chez les femmes, rapportée par les laboratoires du réseau Rénachla, a augmenté ces dernières années ( $2 \%$ en 1999 versus $3,4 \%$ en 2005) [20]. Cette augmentation est en partie liée à la disponibilité de tests de dépistage non invasifs. Cependant, on observe que le nombre de diagnostics positifs a davantage augmenté que le nombre de dépistages $(+135 \%$ versus $+68 \%)$, ce qui suggère une authentique augmentation de l'incidence des infections à Chlamydia chez les femmes. Cette augmentation est observée sur l'ensemble de la France métropolitaine et a notamment concerné des femmes jeunes, âgées de moins de 25 ans. Elles représentent $58 \%$ des chlamydioses identifiées chez les femmes en 2005.

Plusieurs enquêtes de prévalence ont été réalisées entre 2003 et 2005, dans des structures diverses [17]. La prévalence de l'infection à Chlamydia chez les femmes consultant dans les 
centres de planification familiale et les centres d'orthogénie variait de $8 \%$ à $11 \%$. La prévalence chez des étudiantes était nettement moins élevée (entre $1 \%$ et $3 \%$ ), peut-être en raison de comportements à risque moins fréquents qu'en population générale.

Enfin, grâce à l'enquête Contexte de la sexualité en France (CSF) de 2005-2006, on dispose désormais de données de prévalence de l'infection à Chlamydia en population générale [21]. La prévalence est maximale chez les femmes jeunes de 18 à 24 ans $(3,6 \%)$, puis diminue chez les $25-29$ ans $(2,6 \%)$ et est ensuite très faible à partir de 30 ans $(0,5 \%$ chez les $30-44$ ans). La prévalence de l'infection est associée au fait d'avoir eu plusieurs partenaires ou un nouveau partenaire dans les douze derniers mois, mais $44 \%$ des femmes porteuses de l'infection ne présentaient pas cette caractéristique.

\section{Conclusion}

À l'échelle mondiale, environ la moitié des personnes infectées par le VIH sont des femmes et cette proportion atteint presque $60 \%$ en Afrique sub-saharienne. L'Afrique compte plus des trois quarts des femmes séropositives dans le monde. Ailleurs, dans plusieurs zones géographiques où l'épidémie est plus récente, la proportion de femmes augmente encore.

L'épidémiologie des autres maladies sexuellement transmissibles est moins bien connue, mais l'intrication entre celles-ci et l'infection par le VIH est maintenant bien documentée. Les maladies sexuellement transmissibles facilitent la transmission du $\mathrm{VIH}$, en raison d'une plus grande infectiosité en cas de co-infection et d'une plus grande susceptibilité liée à l'inflammation ou aux ulcérations induites. Inversement, traiter une maladie sexuellement transmissible permet de réduire le risque de transmission par le VIH. C'est pourquoi, la lutte contre ce type de maladies doit faire partie intégrante des programmes nationaux de lutte contre le VIH.

En France, les données épidémiologiques montrent aussi une tendance à la féminisation de l'infection à $\mathrm{VIH}$, en raison de la progression de la contamination hétérosexuelle. Cette féminisation semble s'être stabilisée depuis 2002. Les mêmes tendances sont observées au niveau de l'Europe de l'Ouest et les principales caractéristiques de ces femmes y sont assez semblables.

La transmission du VIH concerne une part importante de femmes d'Afrique sub-saharienne, relativement jeunes, contaminées par rapports hétérosexuels et surreprésentées en Île-de-France. Le dépistage de ces femmes se fait rarement dans les mois qui suivent leur contamination, mais se fait aussi rarement au stade tardif de sida, sans doute grâce aux opportunités de dépistage lors des grossesses. On constate de plus une tendance prometteuse dans l'amélioration de l'accès au dépistage, qu'il s'agit bien sûr de poursuivre.
Autour des années 2000, les flux migratoires ont peut-être contribué à une augmentation du nombre de femmes africaines atteintes par le VIH/sida diagnostiquées en France mais, au cours des années plus récentes, leur nombre a diminué alors même que les flux migratoires étaient toujours en augmentation. La part des infections acquises en France par rapport à celles acquises dans les pays d'origine semble avoir tendance à augmenter, ce qui nécessite de ne pas relâcher les efforts de prévention en direction des personnes originaires d'Afrique sub-saharienne.

Le nombre de femmes françaises qui découvrent leur séropositivité a lui aussi tendance à diminuer depuis 2003 , ce qui est plutôt favorable en termes de nouvelles contaminations mais dépend aussi de modifications éventuelles dans l'accès au dépistage, qui sont difficiles à évaluer. La transmission du VIH par rapports hétérosexuels est devenue le mode de contamination quasi exclusif de ces femmes, l'usage de drogues étant désormais très peu contributif.

Presque une femme sur cinq est dépistée à un stade tardif de l'infection (sida), ce qui représente une proportion non négligeable, qui ne diminue pas. Ce constat pourrait s'expliquer par le fait que ces femmes se sentent peu à risque, dans la mesure où elles ont souvent été contaminées par leur partenaire habituel. Même s'il existe un biais par rapport au recueil de cette variable, lié au nombre important de non-réponses, il parait important que les femmes ayant des partenaires multiples ne soient pas la seule cible du dépistage du VIH.

II paraît aussi important de renforcer encore les pratiques de dépistage vis-à-vis des infections à Chlamydia trachomatis chez les femmes jeunes, puisqu'elles sont particulièrement touchées, que l'impact sur la fécondité est loin d'être négligeable et que le traitement est simple.

La recrudescence observée des autres maladies sexuellement transmissibles (syphilis, gonocoques) chez les hommes nécessite aussi d'être vigilant, dans la mesure où ces pathologies peuvent se propager à la population générale et donc aux femmes, à partir d'un groupe d'hommes particulièrement exposés.

\section{Références bibliographiques}

1. OMS. Genre et VIH/sida. www.who.int/gender/hiv_aids/fr

2. Onusida. Le point sur l'épidémie de sida. Décembre 2004.

3. OMS. Essai sur le sulfate de cellulose comme microbicide interrompu. http:// www.who.int/mediacentre/news/statements/2007/s01/fr/index.html

4. Onusida. La coalition mondiale sur les femmes et le sida. http://data.unaids. org/Publications/IRC-pub07/JC1237-GCWA-Prevention-4_fr.pdf

5. Onusida. Le point sur l'épidémie de sida. Décembre 2006.

6. EuroHIV. HIVIAIDS Surveillance in Europe. Mid-year report 2006. Saint-Maurice : Institut de veille sanitaire, $2007, N^{\circ} 74$.

7. EuroHIV. HIVIAIDS Surveillance in Europe. Mid-year report 2005. Saint-Maurice : Institut de veille sanitaire, $2006, N^{\circ} 72$. 
8. Couturier E, Brossard Y, de Benoist AC, Six C, Larsen M, Henrion R, Brunet $J B$. Prévalence de l'infection VIH chez les femmes enceintes de la région parisienne. Une enquête anonyme non corrélée : Prevagest 1991-1993-19951997. Bull Epidemiol Hebd $1998 ; 18: 73-75$.

9. Valin N, Lot F, Larsen C, Gouëzel P, Blanchon T, Laporte A. Parcours sociomédical des personnes originaires d'Afrique subsaharienne atteintes par le VIH, prises en charge dans les hôpitaux d'île-de-France, 2002. Saint-Maurice: InVS, février 2004.

10. Cazein F. Activité de dépistage du VIH. In Lutte contre le VIH/sida et les infections sexuellement transmissibles en France - 10 ans de surveillance, 1996-2005. Saint-Maurice : InVS, mars 2007, p 44-48.

11. ORSîle-de-France. Les connaissances, attitudes, croyances et comportements face au VIH/sida en France. Évolutions 1992-1994-1998-2001-2004. Novembre 2005

12. Le Vu S. Dépistage anonyme et gratuit du VIH. In Lutte contre le VIH/sida et les infections sexuellement transmissibles en France - 10 ans de surveillance, 1996-2005. Saint-Maurice : InVS, mars 2007, 49-57.

13. OMS. Projet de stratégie mondiale de lutte contre les infections sexuellement transmissibles. Rapport du secrétariat. Mai 2006.

14. Del Mar Pujades Rodriguez M, Obasi A, Mosha F, Todd J, Brown D, Changalucha $J$ et al. Herpes simplex virus type 2 infection increases HIV incidence : a prospective study in rural Tanzania. AIDS 2002; 16 : 451-62.
15. Nagot $N$, Ouédraogo A, Foulongne $V$, Konaté I, Weiss HA, Vergne $L$, et al for the ANRS 1285 study group. Reduction of HIV-1 RNA levels with therapy to suppress herpes simplex virus. N Engl J Med $2007 ; 356$; 790-9.

16. Fenton K, Giesecke J, Hamers F. La surveillance européenne des infections sexuellement transmissibles: une initiative opportune et adaptée. Euro Surveill $2001 ; 6(5): 69-70$.

17. Warszawski J, V Goulet. Dépistage systématique des infections à Chlamydia trachomatis : il est temps d'agir. BEH $2006 ; 37-38$ : 275-6.

18. Fenton KA, Lowndes CM. the European surveillance of sexually transmitted infections (ESSTI) network. Recent trends in the epidemiology of sexually transmitted infections in the European Union. Sex Transm Infect $2004 ; 80$ : 255-63.

19. Hughes G, Paine T, Thomas D. Surveillance des infections sexuellement transmissibles en Angleterre et au Pays de Galles. Euro Surveill $2001 ; 6$ : $71-81$.

20. Gallay A, Herida M, Bouyssou-Michel A, GouletV. L'épidémiologie des infections sexuellement transmissibles (hors VIH). In Lutte contre le VIH/sida et les infections sexuellement transmissibles en France - 10 ans de surveillance, 1996-2005. Saint-Maurice : InVS, mars 2007, 66-79.

21. Bajos N. Bozon M, Beltzer N et l'équipe CSF. Contexte de la sexualité en France. Dossier de presse : premiers résultats de l'enquête CSF. Paris, Anrs, Inserm, Ined, 13 mars 2007 (Www.inserm.fr). 